8th Alexander Friedmann International Seminar

on Gravitation and Cosmology

International Journal of Modern Physics: Conference Series

Vol. 3 (2011) 254-265

(C) World Scientific Publishing Company

DOI: $10.1142 /$ S2010194511001346

\title{
AN EARLY UNIVERSE MODEL WITH STIFF MATTER AND A COSMOLOGICAL CONSTANT
}

\author{
G. OLIVEIRA-NETO \\ Departamento de Física, Instituto de Ciências Exatas, \\ Universidade Federal de Juiz de Fora, CEP 36036-330 - Juiz de Fora, MG, Brazil \\ gilneto@fisica.ufj.br \\ G. A. MONERAT, E. V. CORRÊA SILVA, C. NEVES and L. G. FERREIRA FILHO \\ Departamento de Matemática, Física e Computação, Faculdade de Tecnologia, \\ Universidade do Estado do Rio de Janeiro, Rodovia Presidente Dutra, Km 298, \\ Pólo Industrial, CEP 27537-000, Resende-RJ, Brazil. \\ monerat@uerj.br,evasquez@uerj.br,cliffordneves@uerj.br,gonzaga@uerj.br
}

Received 21 June 2011

Revised 23 July 2011

\begin{abstract}
In the present work, we study the quantum cosmology description of a FriedmannRobertson-Walker model in the presence of a stiff matter perfect fluid and a negative cosmological constant. We work in the Schutz's variational formalism and the spatial sections have constant negative curvature. We quantize the model and obtain the appropriate Wheeler-DeWitt equation. In this model the states are bounded therefore we compute the discrete energy spectrum and the corresponding eigenfunctions. In the present work, we consider only the negative eigenvalues and their corresponding eigenfunctions. This choice implies that the energy density of the perfect fluid is negative. A stiff matter perfect fluid with this property produces a model with a bouncing solution, at the classical level, free from an initial singularity. After that, we use the eigenfunctions in order to construct wave packets and evaluate the time-dependent expectation value of the scale factor. We find that it oscillates between maximum and minimum values. Since the expectation value of the scale factor never vanishes, we confirm that this model is free from an initial singularity, also, at the quantum level.
\end{abstract}

Keywords: Stiff matter; Wheeler-DeWitt equation; negative cosmological constant.

PACS numbers: 04.40.Nr,04.60.Ds,98.80.Qc

\section{Introduction}

The great importance of cosmological models where the matter content is represented by a stiff matter perfect fluid was recognized since its introduction by Zeldovich. $^{1,2}$ This perfect fluid has an equation of state of the form, $p=\alpha w$, with $\alpha=1$, where $w$ and $p$ are, respectively, the fluid energy density and pressure. It can also be described by a massless free scalar field. In order to understand better the importance of this perfect fluid for cosmology, one has to compute its energy 
density. In the temporal gauge $(N(t)=1)$, this quantity is proportional to $1 / a(t)^{6}$, where $N(t)$ is the lapse function and $a(t)$ is the scale factor. On the other hand, in the same gauge, the energy density of a radiative perfect fluid is proportional to $1 / a(t)^{4}$. This result indicates that there may have existed a phase earlier than that of radiation, in our Universe, which was dominated by stiff matter. Due to that importance, many physicists have started to consider the implications of the presence of a stiff matter perfect fluid in FRW cosmological models. The first important implication of the presence of stiff matter in FRW cosmological models is in the relic abundance of particle species produced after the 'Big Bang' due to the expansion and cooling of our Universe. ${ }^{3-8}$ The presence of stiff matter in FRW cosmological models may also help explaining the baryon asymmetry and the density perturbations of the right amplitude for the large scale structure formation in our Universe. ${ }^{9,10}$ It may also play an important role in the spectrum of relic gravity waves created during inflation. ${ }^{11}$ Since there may have existed a phase earlier than that of radiation which was dominated by stiff matter some physicists considered quantum cosmological models with this kind of matter. ${ }^{12-14}$

In the present work, we study the quantum cosmology description of a Friedmann-Robertson-Walker (FRW) model in the presence of a stiff matter perfect fluid and a negative cosmological constant. The model has constant negatively curved spatial sections. We work in the Schutz's variational formalism. ${ }^{15,16} \mathrm{We}$ quantize the model and obtain the appropriate Wheeler-DeWitt equation. In this model the states are bounded therefore we compute the discrete energy spectrum and the corresponding eigenfunctions. In the present work, we consider only the negative eigenvalues and their corresponding eigenfunctions. This choice implies that the energy density of the perfect fluid is negative. A stiff matter perfect fluid with this property has already been considered in the literature. ${ }^{17-19}$ It produces a model with a bouncing solution, at the classical level, free from an initial singularity. After that, we use the eigenfunctions in order to construct wave packets and evaluate the time-dependent expectation value of the scale factor. We find that it oscillates between maximum and minimum values. Since the expectation value of the scale factor never vanishes, we confirm that this model is free from an initial singularity, also, at the quantum level.

The presence of a negative cosmological constant in the present model implies that the universe described by it has a maximum size, in other words it is bounded. Taking into account the current cosmological observations, a negative cosmological constant will not be able to describe the present accelerated expansion of our Universe. It is not our intention to describe the present state of our Universe with this model. On the other hand, it is our intention to describe a 'possible' state of our primordial Universe. One important theory which is a strong candidate to describe the unification of all known physical interactions is superstring theory. ${ }^{20,21}$ Due to that, many physicists believe that superstring theory will correctly describe the quantum gravity effects that took place at the beginning of our Universe. There is 
an important conjecture which tells that Type IIB string theory on $\left(A d S_{5} \times S_{5}\right)_{N}$ plus some appropriate boundary conditions is dual to $N=4 d=3+1 U(N)$ superYang-Mills. ${ }^{22}$ It means that, possibly, for an appropriate description of the known physical interactions through superstring theories, the strings have to exist in an Anti-DeSitter spacetime. The Anti-DeSitter spacetime has a negative cosmological constant, therefore it seems worthwhile to study spacetimes with a negative cosmological constant if one wants to understand more about a 'possible' initial state of our primordial Universe. Of course, after that initial state the Universe would have to undergo a transition where the cosmological constant would change sign. Besides that, several important theoretical results and predictions in quantum cosmology have been obtained with a negative cosmological constant. ${ }^{23-26}$ Considering a subset of all four-dimensional spacetimes with constant negative curvature and compact space-like hypersurfaces, S. Carlip et al showed how to compute the sum over topologies leading to the no-boundary wave-function. ${ }^{23,24}$ These spacetimes are curved only due to the presence of a negative cosmological constant. In Ref. 23 it was shown how to obtain a vanishing cosmological constant as a prediction from the no-boundary wave-function and in Ref. 24 it was shown how to obtain predictions about the topology of the Universe from the no-boundary wave-function. We may also mention the result in Ref. 25, where the WKB no-boundary wave-function of a homogeneous and isotropic Universe with a negative cosmological constant was computed. Due to the regularity condition imposed upon the spacetimes contributing to the no-boundary wave-function, it was shown that only a well defined, discrete spectrum for the cosmological constant is possible. It was also found that among the spacetimes contributing to the wave function, there were two complex conjugate ones that showed a new type of signature change.

The present paper is organized as follows. In Sec. 2, we introduce the classical model and obtain the appropriate Friedmann equation. With the aid of the potential curve coming from this equation, we comment on the general behavior of the classical solutions. In Sec. 3, we quantize the model by solving the corresponding Wheeler-DeWitt equation. The wave-function depends on the scale factor $a$ and on the canonical variable associated to the fluid, which in the Schutz variational formalism plays the role of time $(T)$. We separate the wave-function in two parts, one depending solely on the scale factor and the other depending only on the time. The solution in the time sector of the Wheeler-DeWitt equation is trivial, leading to an imaginary exponential of the type $e^{-i E \tau}$, where $E$ is the system energy and $\tau=-T$. The scale factor sector of the Wheeler-DeWitt equation gives rise to an eigenvalue equation. We find approximate solutions. In Sec. 4, we construct wave packets from the eigenfunctions and compute the time-dependent, expectation value of the scale factor. We find that the expectation value of the scale factor shows bounded oscillations. Since the expectation value of the scale factor never vanishes, we confirm that this model is free from a big bang singularity, also, at the quantum level. Finally, in Sec. 5, we summarize the main points and results of our paper. 


\section{The Classical Model}

The present Friedmann-Robertson-Walker cosmological model is characterized by the scale factor $a(t)$ and has the following line element,

$$
d s^{2}=-N^{2}(t) d t^{2}+a^{2}(t)\left(\frac{d r^{2}}{1+r^{2}}+r^{2} d \Omega^{2}\right),
$$

where $d \Omega^{2}$ is the line element of the two-dimensional sphere with unitary radius, $N(t)$ is the lapse function and we are using the natural unit system, where $\hbar=c=$ $8 \pi G=1$. In this model the spatial sections are some closed three-dimensional solid with negative constant curvature, locally isometric ${ }^{27}$ to $H^{3}$. The matter content of the model is represented by a perfect fluid with four-velocity $U^{\mu}=\delta_{0}^{\mu}$ in the comoving coordinate system used, plus a negative cosmological constant $(\Lambda)$. The total energy-momentum tensor is given by,

$$
T_{\mu \nu}=(w+p) U_{\mu} U_{\nu}-p g_{\mu \nu}-\Lambda g_{\mu \nu} .
$$

As mentioned above, here, we assume that $p=w$, which is the equation of state for stiff matter.

Einstein's equations for the metric (1) and the energy momentum tensor (2) are equivalent to the Hamilton's equations generated by the total Hamiltonian $N(t) \mathcal{H}$, where,

$$
\mathcal{H}=-\frac{p_{a}^{2}}{12 a}+3 a+\Lambda a^{3}+\frac{p_{T}}{a^{3}} .
$$

The variables $p_{a}$ and $p_{T}$ are the momenta canonically conjugated to the variables $a$ and $T$, respectively.

The classical dynamics is governed by the Hamilton's equations, derived from the total Hamiltonian $N(t) \mathcal{H}$ Eq. (3). In the gauge $N(t)=a(t)$, they are,

$$
\left\{\begin{array}{l}
\dot{a}=\frac{\partial N \mathcal{H}}{\partial p_{a}}=-\frac{p_{a}}{6} \\
\dot{p_{a}}=-\frac{\partial N \mathcal{H}}{\partial a}=-6 a-4 \Lambda a^{3}+2 \frac{p_{T}}{a^{3}} \\
\dot{T}=\frac{\partial N \mathcal{H}}{\partial p_{T}}=\frac{1}{a^{2}} \\
\dot{p_{T}}=-\frac{\partial N \mathcal{H}}{\partial T}=0 .
\end{array}\right.
$$

Here, the dot means derivative with respect to the conformal time $\tau \equiv N t$, which in the present gauge is equal to at.

We also have the constraint equation $\mathcal{H}=0$. It gives rise to the Friedmann equation,

$$
\dot{a}^{2}+V_{c}(a)=0
$$

where the potential $V_{c}(a)$ is equal to,

$$
V_{c}(a)=-a^{2}-\frac{\Lambda a^{4}}{3}-\frac{p_{T}}{3 a^{2}} .
$$




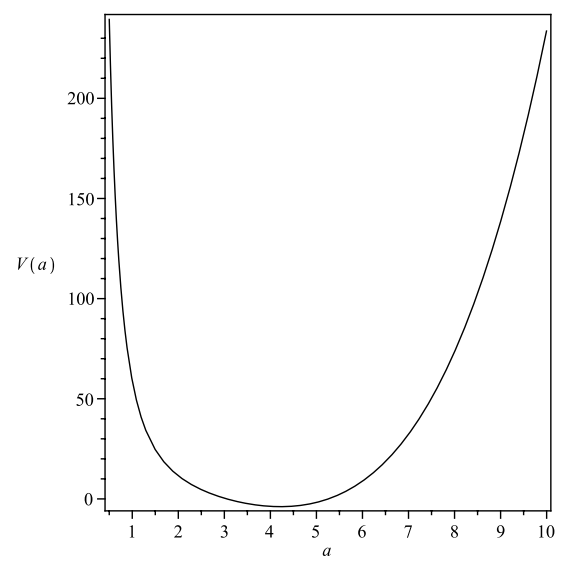

Fig. 1. $V_{c}(a)$ for $\Lambda=-0.1$ and $p_{T}=-180$.

For the present situation where $\Lambda<0$, we have bounded solutions. The classical solutions are bouncing ones, free from an initial singularity, because we are considering a stiff matter perfect fluid with negative energy density. Those results can be directly seen from the potential expression. A particular example of $V_{c}(a)$, for $\Lambda=-0.1$ and $p_{T}=-180$, is given in Fig. 1 .

\section{The Quantum Model}

We wish to quantize the model following the Dirac formalism for quantizing constrained systems. ${ }^{28-31}$ First we introduce a wave-function which is a function of the canonical variables $\hat{a}$ and $\hat{T}$,

$$
\Psi=\Psi(\hat{a}, \hat{T}) .
$$

Then, we impose the appropriate commutators between the operators $\hat{a}$ and $\hat{T}$ and their conjugate momenta $\hat{p}_{a}$ and $\hat{p}_{T}$. Working in the Schrödinger picture, the operators $\hat{a}$ and $\hat{T}$ are simply multiplication operators, while their conjugate momenta are represented by the differential operators

$$
\hat{p}_{a} \rightarrow-i \frac{\partial}{\partial a}, \quad \hat{p}_{T} \rightarrow-i \frac{\partial}{\partial T} .
$$

Finally, we demand that $\hat{\mathcal{H}}$, the superhamiltonian operator corresponding to (3), annihilate the wave-function $\Psi$ Eq. (7), which leads to Wheeler-DeWitt equation

$$
\left(-\frac{1}{12} \frac{\partial^{2}}{\partial a^{2}}-\left(3 a^{2}+\Lambda a^{4}\right)\right) \Psi(a, \tau)=i \frac{1}{a^{2}} \frac{\partial}{\partial \tau} \Psi(a, \tau)
$$

where the new variable $\tau=-T$ has been introduced.

The operator $\hat{\mathcal{H}}$ is self-adjoint ${ }^{32}$ with respect to the internal product,

$$
(\Psi, \Phi)=\int_{0}^{\infty} d a \frac{1}{a^{2}} \Psi(a, \tau)^{*} \Phi(a, \tau),
$$


if the wave-functions are restricted to the set of those satisfying either $\Psi(0, \tau)=0$ or $\Psi^{\prime}(0, \tau)=0$, where the prime / means the partial derivative with respect to $a$.

The Wheeler-DeWitt equation (9) may be solved by writing $\Psi(a, \tau)$ as,

$$
\Psi(a, \tau)=e^{-i E \tau} \eta(a)
$$

where $\eta(a)$ depends solely on $a$. Then $\eta(a)$ satisfies the eigenvalue equation

$$
-\frac{d^{2} \eta(a)}{d a^{2}}+V(a) \eta(a)=12 E \frac{1}{a^{2}} \eta(a),
$$

where the potential $V(a)$ is given by

$$
V(a)=-36 a^{2}-12 \Lambda a^{4} .
$$

In the same way as in the classical regime, the potential $V(a)$ Eq. (13) gives rise to bound states. Therefore, the energies $E$, Eq. (12), of those states will form a discrete set of eigenvalues $E_{n}$, where $n=1,2,3, \ldots$. For each eigenvalue $E_{n}$ there will be a corresponding eigenvector $\eta_{n}(a)$. The general solution to the Wheeler-DeWitt equation (9) is a linear combination of all those eigenvectors,

$$
\Psi(a, \tau)=\sum_{n=1}^{\infty} C_{n} \eta_{n}(a) e^{-i E_{n} \tau},
$$

where $C_{n}$ are free coefficients to be specified.

We are going to use the Galerkin or spectral method (SM), in order to solve the eigenvalue equation (12). This method is well presented in Ref. 33 and it has already been used in quantum cosmology. ${ }^{34,35}$ In the SM, one must choose orthonormal basis functions in order to expand the solution to the eigenvalue equation. The solutions to the present eigenvalue equation (12) must fall sufficiently fast for large scale factor values $(a)$. It means that we must restrict the initial infinity domain of our variable $a$, to a finite domain. Say, $0<a<L$, where $L$ is a finite number that has to be fixed. Here, we shall consider solutions satisfying the condition $\Psi(0, \tau)=0$. Putting together all the above properties of the solutions to Eq. (12), it is convenient (but not mandatory) to choose our basis functions to be sine functions. Therefore, we may write $\eta_{n}(a)$ in Eq. (12) as,

$$
\eta_{n}(a)=\sum_{n=1}^{\infty} A_{n} \sqrt{\frac{2}{L}} \sin \left(\frac{n \pi a}{L}\right),
$$

where the $A_{n}$ 's will be determined by the SM. In the same $a$ domain, we may also expand, in the same basis, the other two important functions of $a$ appearing in Eq. (12),

$$
\begin{aligned}
& V(a) \eta_{n}(a)=\sum_{n=1}^{\infty} B_{n} \sqrt{\frac{2}{L}} \sin \left(\frac{n \pi a}{L}\right), \\
& \left(\frac{12}{a^{2}}\right) \eta_{n}(a)=\sum_{n=1}^{\infty} B_{n}^{\prime} \sqrt{\frac{2}{L}} \sin \left(\frac{n \pi a}{L}\right),
\end{aligned}
$$


where $V(a)$ is given in Eq. (13) and the coefficients $B_{n}$ and $B_{n}^{\prime}$ can be easily determined. They are determined with the aid of Eq. (15) and the fact that the basis functions are orthonormal. The coefficients $B_{n}$ and $B_{n}^{\prime}$ are given by,

$$
\begin{aligned}
& B_{n}=\sum_{m=1}^{\infty} C_{m, n} A_{m}, \\
& B_{n}^{\prime}=\sum_{m=1}^{\infty} C_{m, n}^{\prime} A_{m},
\end{aligned}
$$

where,

$$
\begin{aligned}
C_{m, n} & =\frac{2}{L} \int_{0}^{L} \sin \left(\frac{m \pi a}{L}\right) V(a) \sin \left(\frac{n \pi a}{L}\right) d a, \\
C_{m, n}^{\prime} & =\frac{2}{L} \int_{0}^{L} \sin \left(\frac{m \pi a}{L}\right)\left(\frac{12}{a^{2}}\right) \sin \left(\frac{n \pi a}{L}\right) d a .
\end{aligned}
$$

Introducing the results Eqs. (15)-(21) in the eigenvalue equation (12) and using the fact that the basis functions are orthonormal, we obtain,

$$
\left(\frac{n \pi}{L}\right)^{2} A_{n}+\sum_{m=1}^{\infty} C_{m, n} A_{m}=E \sum_{m=1}^{\infty} C_{m, n}^{\prime} A_{m} .
$$

In order to derive some numerical results we must fix a finite number of basis functions, in other words, a finite number for the maximum value of the summation indices. Let us call this number $N$. The greater the value of $N$, the closer our results will be to the exact ones. We shall be restricted by our computational resources. Equation (22), may be written in a compact notation as,

$$
D^{\prime-1} D A=E A
$$

where $D$ and $D^{\prime}$ are $N \times N$ square matrices and their elements are obtained from Eq. (22). The solution to Eq. (23) gives the eigenvalues and corresponding eigenfunctions to the bound states of our quantum cosmology model.

It is important to mention that the most correct form of $\eta(a)$ in the limit when $a \rightarrow 0$ is not given by Eq. (15). In order to obtain that expression for $\eta(a)$, one has to introduce the ansatz $\eta(a)=C a^{\alpha}$ (where $C$ is a constant and $\alpha$ is a number to be determined) in Eq. (12). After that, one has to discard the terms which have as coefficients the cosmological constant $(\Lambda)$ and the curvature of the spatial sections $(k=-1)$. The main motivation to discard those terms is that they are proportional to $a^{\alpha+4}$ and $a^{\alpha+2}$ and should be less important, in the limit $a \rightarrow 0$, than the term whose coefficient is an algebraic equation for $\alpha$. Finally, one solves the resulting equation imposing that the coefficient of the only remaining term, proportional to $a^{\alpha-2}$, vanishes. This gives rise to a second order algebraic equation for $\alpha$, which solution satisfying the boundary condition $\eta(0)=0$ is given by: $\alpha=$ 
$0.5+\sqrt{0.25+12|E|}$. Therefore, the most correct form for $\eta(a)$ in the limit when $a \rightarrow 0$ is given by,

$$
\eta(a)=C a^{0.5+\sqrt{0.25+12|E|}} .
$$

We notice that it cannot represent the correct expression for $\eta(a)$ in the limit $a \rightarrow \infty$ because it diverges in that limit. That solution is already known in the literature. ${ }^{36}$

\section{Energy Spectrum, Wave Packet and Mean Value.}

In this section we solve Eq. (12) using the SM. In order to choose the number of basis functions $N$ and the values of $\Lambda$, we performed the following numerical procedures. First of all, in order to choose the values of $\Lambda$, we solved numerically, the eigenvalue equation (23) for several different values of $\Lambda$ and fixed values of $N$ and $L$. We noticed that, although, we are free to choose any value of $\Lambda$, the results accuracy for small absolute values of $\Lambda$ is better than for large absolute values of $\Lambda$, for a given number of basis functions $N$. This means that, if we use a large absolute value of $\Lambda$, we need to increase $N$ to obtain the same accuracy of the case with a small absolute value of $\Lambda$. This, of course, would increase the computation time. Therefore, we have decided to use small absolute values of $\Lambda$. Taking in account this considerations, we choose $\Lambda=-0.1$ in the rest of our paper. In order to choose the value of $N$, we solved numerically, the eigenvalue equation (23) for several different values of $N$ and fixed values of $\Lambda$ and $L$. Then, we compared the eigenvalues coming from the different choices of $N$. We noticed that only some of the first eigenvalues, for each different choice of $N$, remained the same up to a satisfactory accuracy. Therefore, we have decided to use $N=100$ and take only the first eighteen eigenvalues to construct the wave packet. The accuracy of the eigenvalues, in this case, is in the tenth digit after the dot. Finally, it is important to mention that based on comparisons with results of other models studied with the $\mathrm{SM},{ }^{35}$ we chose $L=6$, in the present case.

Now, using all these values: $N=100, \Lambda=-0.1$ and $L=6$, in the determinant constructed from Eq. (23), we solve it and obtain the first 100 energy eigenvalues for the present case. From those, we take the first 18 energy levels and list them in Table 1 .

In order to give an idea how the energy spectrum depends on $\Lambda$, we have constructed the curve of the fundamental energy level $E_{1}$ versus $\Lambda$. It is given in Fig. 2 . We notice that $E_{1}$ decreases when one increases $\Lambda$.

Table 1. The eighteen lowest energy levels for a FRW model with $k=-1, \Lambda=-0.1$, a stiff matter perfect fluid $(p=\rho), N=100$ and $L=6$.

\begin{tabular}{|c|c|c|}
\hline$E_{1}=-380.2201284331828$ & $E_{2}=-342.1147751350869$ & $E_{3}=-305.9147225014253$ \\
\hline$E_{4}=-271.6319016521779$ & $E_{5}=-239.2791871064332$ & $E_{6}=-208.8705235210961$ \\
\hline$E_{7}=-180.4210774184946$ & $E_{8}=-153.9474207233139$ & $E_{9}=-129.4677552918257$ \\
\hline$E_{10}=-107.0021912238143$ & $E_{11}=-86.57309703051190$ & $E_{12}=-68.20554802882278$ \\
\hline$E_{13}=-51.92791264850499$ & $E_{14}=-37.77263895532904$ & $E_{15}=-25.77734426711615$ \\
\hline$E_{16}=-15.98638945709977$ & $E_{17}=-8.453288958554614$ & $E_{18}=-3.244733126937446$ \\
\hline
\end{tabular}




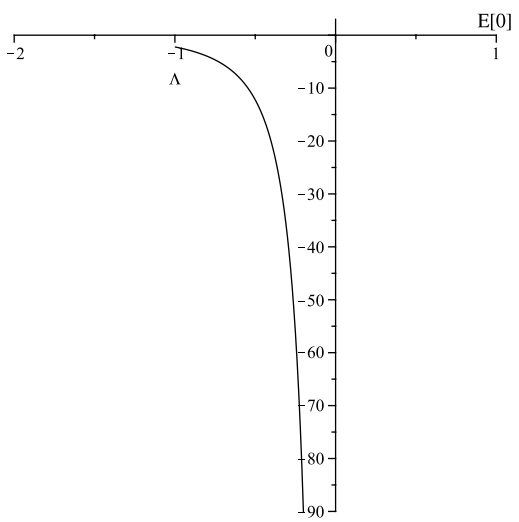

Fig. 2. Dependence of $E_{1}$ with $\Lambda$, for $N=100$ and $L=6$.

It is important to mention that even though the expression of $\eta(a)$ given by Eq. (15) is not the most correct one, in the limit $a \rightarrow 0$, we were able to determine numerically that it converges rapidly to zero in that limit. In particular, for the first energy level the corresponding eigenfunction oscillations about the zero value, in a region very close to it $(0 \leq a \leq 0.2)$, are of the order of $10^{-12}$. For the eighteenth energy level the corresponding eigenfunction oscillations about the zero value, in a region very close to it $(0 \leq a \leq 0.04)$, are of the order of $10^{-9}$. For the other eigenfunctions the oscillations about zero, in regions very close to zero, have values between the ones given above.

Next, we construct the wave packet $\Psi(a, \tau)$ Eq. (14), with the aid of $\eta_{n}(a)$ Eq. (15) and the energy levels in Table 1. Our numerical study showed that, the energy eigenfunctions in the linear combination Eq. (14) are orthonormal and $\Psi(a, \tau)$ Eq. (14), has a constant norm. In the linear combination Eq. (14), we set $C_{n}$ equal to one, for the first eighteen values of $n$ and $C_{n}$ equal to zero for the other values of $n$. In Fig. 3, we show, as an example, the modulus squared of a wave packet

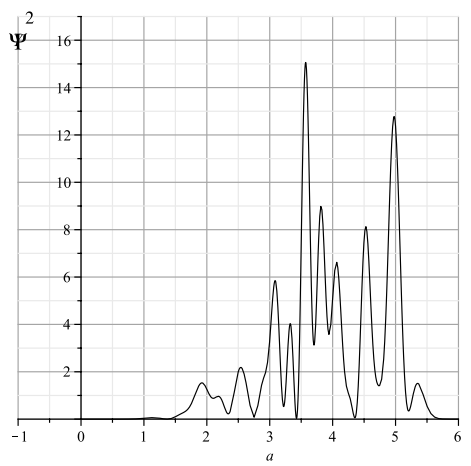

Fig. 3. Modulus squared of a wave packet constructed with the first eighteen energy levels for $\tau=1000, L=6$ and $\Lambda=-0.1$. 


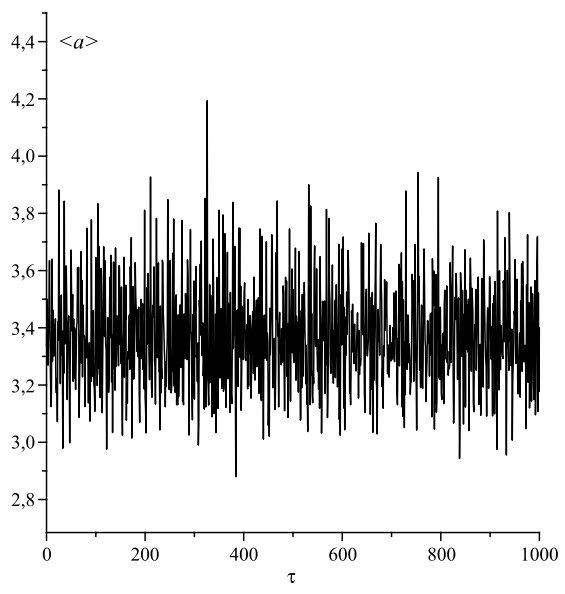

Fig. 4. $\langle a\rangle$ computed with the wave packet constructed with the first eighteen energy levels for the period from $\tau=0$ until $\tau=1000, L=6$ and $\Lambda=-0.1$.

constructed with the first eighteen energy levels, given in Table 1, for $\tau=1000$, $L=6$ and $\Lambda=-0.1$.

Finally, using the wave packet $\Psi(a, \tau)$ we compute the mean value for the scale factor $a$, according to the following expression,

$$
\langle a\rangle(\tau)=\frac{\int_{0}^{\infty} a^{-1}|\Psi(a, \tau)|^{2} d a}{\int_{0}^{\infty} a^{-2}|\Psi(a, \tau)|^{2} d a} .
$$

We computed this quantity for many different time intervals. For all this different values, we observed that $\langle a\rangle$ performs bounded oscillations and never assume the zero value. Therefore, we confirm that these models are free from singularities, also, at the quantum level. As an example, we show in Fig. 4 the mean value computed with the wave packet constructed with the first eighteen energy levels, given in Table 1 , for the interval from $\tau=0$ until $\tau=1000, L=6$ and $\Lambda=-0.1$.

\section{Conclusions.}

In the present paper, we quantized a Friedmann-Robertson-Walker model in the presence of a stiff matter perfect fluid and a negative cosmological constant. We used the variational formalism of Schutz. The model has spatial sections with negative constant curvature. The quantization of the model gave rise to a Wheeler-DeWitt equation, for the scale factor. We found the approximate eigenvalues and eigenfunctions of that equation by using the Galerkin or spectral method. In the present work, we considered only the negative energy eigenvalues and their corresponding eigenfunctions. This choice implies that the energy density of the perfect fluid is negative. A stiff matter perfect fluid with this property produces a model with a bouncing solution, at the classical level, free from an initial singularity. After that, we used the eigenfunctions in order to construct wave packets and evaluate the time 
dependent, expected value of the scale factor. We found that the expected value of the scale factor evolve with bounded oscillations. Since the expectation value of the scale factor never vanish, we confirm that this model is free singularities, also, at the quantum level.

\section{Acknowledgments}

E. V. Corrêa Silva (Researcher of CNPq, Brazil), G. A. Monerat, G. Oliveira-Neto, C. Neves and L. G. Ferreira Filho thank CNPq and FAPERJ for partial financial support. G. Oliveira-Neto thanks FAPEMIG for partial financial support.

\section{References}

1. Y. B. Zeldovich, Zh. Eksp. Teor. Fiz. 41, 1609 (1961).

2. Y. B. Zeldovich, Sov. Phys. JETP 14, 1143 (1962).

3. M. Kamionkowski and M. S. Turner, Phys. Rev. D 42, 3310 (1990).

4. M. Joyce, Phys. Rev. D 55, 1875 (1997).

5. P. Salati, Phys. Lett. B 571, 121 (2003).

6. C. Pallis, Nucl. Phys. B 751, 129 (2006).

7. M. E. Gomez, S. Lola, C. Pallis and J. Rodriguez-Quintero, JCAP 01, 027 (2009).

8. C. Pallis, Nucl. Phys. B 831, 217 (2010).

9. Y. B. Zeldovich, MNRAS 160, 1 (1972).

10. M. Joyce and T. Prokopec, Phys. Rev. D 57, 6022 (1998).

11. V. Sahni, M. Sami and T. Souradeep, Phys. Rev. D 65, 023518 (2001).

12. R. Colistete, Jr., J. C. Fabris and N. Pinto-Neto, Phys. Rev. D 57, 4707 (1998).

13. R. Colistete, Jr., J. C. Fabris and N. Pinto-Neto, Phys. Rev. D 62, 083507 (2000).

14. F. T. Falciano, N. Pinto-Neto and E. S. Santini, Phys. Rev. D 76, 083521 (2007).

15. B. F. Schutz, Phys. Rev. D 2, 2762 (1970).

16. B. F. Schutz, Phys. Rev. D 4, 3559 (1971).

17. P. Peter and N. Pinto-Neto, Phys. Rev. D 66, 063509 (2002).

18. F. Finelli, JCAP 0310, 011 (2003).

19. F. Finelli, P. Peter and N. Pinto-Neto, Phys. Rev. D 77, 103508 (2008).

20. M. B. Green, J. H. Schwarz and E. Witten, Superstring theory, Vol. 1 (Cambridge University Press, Cambridge, 1987).

21. M. B. Green, J. H. Schwarz and E. Witten, Superstring theory, Vol. 2 (Cambridge University Press, Cambridge, 1987).

22. J. Maldacena, Adv. Theor. Math. Phys. 2, 231 (1998).

23. S. Carlip, Phys. Rev. Lett. 79, 4071 (1997).

24. M. Anderson, S. Carlip, J. G. Ratcliffe, S. Surya and S. T. Tschantz, Class. Quant. Grav. 21, 729 (2004).

25. G. Oliveira-Neto, Phys. Rev. D 58, 10750 (1998).

26. G. A. Monerat, E. V. Corrêa Silva, G. Oliveira-Neto, L. G. Ferreira Filho and N. A. Lemos, Phys. Rev. D 73, 044022 (2006).

27. W. P. Thurston, The Geometry and Topology of Three-Manifolds (Princeton University Press, Princeton, 1982).

28. P. A. M. Dirac, Can. J. Math. 2, 129 (1950).

29. P. A. M. Dirac, Proc. Roy. Soc. London A 249, 326 (1958).

30. P. A. M. Dirac, Proc. Roy. Soc. London A 249, 333 (1958).

31. P. A. M. Dirac, Phys. Rev. 114, 924 (1959). 
32. N. A. Lemos, J. Math. Phys. 37, 1449 (1996).

33. J. P. Boyd, Chebyshev and Fourier Spectral Methods, 2nd edn. (Dover, New York, 2001).

34. P. Pedram, M. Mirzaei and S. S. Gousheh, Comput. Phys. Commun. 176, 581 (2007).

35. P. Pedram, M. Mirzaei, S. Jalalzadeh and S. S. Gousheh. Gen. Rel. Grav. 40, 1663 (2008).

36. F. G. Alvarenga, J. C. Fabris, N. A. Lemos and G. A. Monerat, Gen. Rel. Grav. 34, 651 (2002). 